\title{
Alzheimer's disease: neurobiological advances supporting proposals for new therapeutical approaches
}

\author{
André Nieoullon \\ Cellular Interactions, Neurodegeneration and Neuroplasticity Research Unit, UMR6186-CNRS \\ and l'Université de la Méditerranée, Marseille, France
}

Received $21^{\text {st }}$ April 2004.

Revised $6^{\text {th }}$ May 2004.

Published online $4^{\text {th }}$ June 2004.

\begin{abstract}
Summary
Alzheimer's disease is one of the most important diseases related to ageing. The social and human cost is very high especially because of the increase in the normal life span in developed countries. There is presently no cure for the disease and stimulating the cholinergic central neurotransmission is today the only therapeutic approach which has been shown to successfully improve the cognitive state in a significant population of patients. In such a context, research projects should be aimed specifically at reducing brain lesions resulting from the disease, especially those due to the amyloid peptide accumulation, and at reducing or even halting the clinical evolution of the disease and consequent neurodegenerative processes.
\end{abstract}

Keywords: neuroprotection - amyloïd protein precursor (APP) - $\beta$ A4 protein - tau protein astrocytes - excitatory amino acids

\section{INTRODUCTION}

In spite of the considerable amount of new clinical and experimental data, for example in the field of genetic approaches to the disease, it is worth emphasising that recent cellular and molecular investigations have unfortunately not provided any major conceptual advances leading to reconsideration of the treatment of Alzheimer's disease. Basically, stimulation of the cholinergic central neurotransmission remains today the only therapeutic approach which has been shown to successfully improve the cognitive state in a significant population of patients. Thus, curing the disease still remains an unrealistic prospect. However, many research groups actively contribute to the development of putative new therapeutic strategies aimed at simply delaying the course of such a major neurodegenerative disease. Indeed, since Alzheimer's disease is primarily related to ageing it is interesting to note that just delaying the course of the disease for some years could actually contribute to the reduction of the number of patients and, further, to the social cost of the increase in the life span measured each year in developed countries.

Consequently, even if curing the disease still remains a major societal aim, therapeutic approaches could more realistically focus on symptomatic treatments, with the double goal of improving available medicines and of introducing efficient neuroprotective agents able to delay the course of the disease or its clinical onset. In this respect, research projects should be aimed firstly, at contributing to improved stimulation of cholinergic transmission and available drugs; secondly, at specifically reducing brain lesions resulting from the disease, specially due to amyloïd protein 
accumulation; and thirdly, at reducing or even stopping the clinical evolution of the disease and consequent neurodegenerative processes. In the last case, however, putative neuroprotective strategies have to be directed to the cellular and molecular mechanisms of neuronal death, even if they are far from being fully characterised as Alzheimer's disease. Finally, another possibility in the future would be to propose alternative strategies for brain repair. These last proposals are very stimulating and the characterisation of stem cells, which may or may not be transfected before transplantation, theoretically represents a new therapeutic approach to be actively investigated before use on humans is proposed.

\section{IMPROVING THE DEFICIENT CHOLINERGIC TRANSMISSION IN THE BRAIN}

Impaired cholinergic transmission in Alzheimer's disease has been characterised for a longer time. Such a cholinergic deficit was specially evidenced at the cortical level mainly involving neuronal projection from the basal nucleus of Meynert. In addition to tacrine, the clinical use of new generations of acetylcholinesterase inhibitors such as donepezil, rivastigmine, and galantamine, etc., which have reduced side effects, presently results in the satisfactory improvement of cognitive and social performance for a majority of patients, especially in the earlier stages of the disease (Allain et al. 2002, Cutler and Sramek 2001). These compounds are now largely available world wide, although rather expensive.

A second possibility, however, is to directly stimulate cholinergic transmission using specific receptor agonists. In Alzheimer's disease most of the cholinergic receptors lost in the brain areas related to cognitive functions are represented either by the M2 muscarinic receptor subtype or the nicotinic receptors, especially those containing $\alpha 4$ and $\alpha 7$ sub-units in their structure, which are primarily pre-synaptically located on the degenerating nerve terminals. The M1 receptors are considered as preserved during the course of the disease. Indeed, drugs such as arecoline, milameline or xanomeline, which primarily act as muscarinic M1 receptor agonists, have been used in patients but the clinical efficiency is still being discussed (Emilien et al. 2000, Sramek and Cutler 1999). For example, arecoline has been shown to improve memory processes but with large inter-subject variability. Milameline was poorly tolerated and xanomeline showed improved cognition in patients with the disease.
Compounds acting at the nicotinic receptor sites could be more promising since molecular biology showed that there is a great tissue selectivity in the expression of some of the subunits forming the receptor, as emphasised above. For example, cortical and hippocampal structures were shown to primarily express $\alpha 4$ and $\alpha 7$ subunits. Such subunits may thus contribute to form particular nicotinic receptor subtypes, which could exhibit specific pharmacological and functional properties, leading to a further original pharmacological approach. However, a key problem with compounds such as nicotine could be their addictive properties and possible desensitisation of the over-stimulated receptors. Interestingly, the acetylcholinesterase inhibitor galantamine was shown, besides its inhibitory influence on the acetylcholine degrading enzyme, to potentiate central nicotinic receptor stimulation through an original allosteric modulatory mechanism, which has the great advantage of preventing the desensitisation processes.

Numerous studies have been developed in this way to improve brain cholinergic transmission but it is worth mentioning that such an approach to Alzheimer's disease is highly reductionistic, regarding, for example, the extent of brain cortical and hippocampal lesions, which overpass the cholinergic neurones. However, because stimulation of the cholinergic transmission by means of pre-synaptic mechanisms could also influence other central neuromodulatory systems of cognitive functions, such as dopaminergic, serotoninergic and glutamatergic transmission, for example, and also improve metabolism in the forebrain, stimulating the cholinergic transmission using pharmacological compounds, could result in large behavioural stimulation, including more active attentional processes and general cognitive functions. Consequently, stimulating cholinergic transmission, primarily by using inhibitors of acetylcholinesterases, is presently the only possibility of improving the cognitive status of patients during the course of the disease.

\section{LIMITING THE DEVELOPMENT OF BRAIN LESIONS IN ALZHEIMER'S DISEASE}

In Alzheimer's disease, three main types of lesions have been characterised: the accumulation of the amyloid protein known as $\beta \mathrm{A} 4$ protein, the formation of paired neurofibrarilly tangles, in which the tau protein is a major component, and an inflammatory process correlated to glial reaction involving microglial proliferation and hypertrophy of a subpopulation of astrocytes. However, although generally accepted, the specificity of the lesions 
relating to the disease and the relationship between the extent of tissue damage and behavioural and cognitive impairments still remains to be definitively demonstrated. To our knowledge, attempts have been made only to limit $\beta A 4$ toxicity in the brain. Three further main objectives have been proposed: to limit peptide production; to reduce its aggregation which is supposed to induce brain lesion; and to dissolve any present peptide aggregates.

Present knowledge of the metabolism of the amyloid protein and of its related metabolic precursor called the amyloid precursor protein (APP) has led to proposals for pharmacological strategies aimed at blocking the pathological biosynthesis of the $\beta A 4$ peptide, which involves $\beta$-secretases ( $\beta$-site APP cleaving enzyme or BACE) or $\gamma$-secretases, the activity of which is modulated by presenilins, compared to normal processing, which involves $\alpha$-secretases submitted to the regulatory influence of the protein kinase $\mathrm{C}$ (PKC). In order to limit $\beta \mathrm{A} 4$ biosynthesis and accumulation, four main strategies have been proposed (Citron 2002, Gandy 2002, Scorer 2001):

(i) The use of vaccination using $\beta \mathrm{A} 4$ synthetic peptide: this strategy is controversial but still remains an open possibility (Hock et al. 2003) in spite of major side effects recently reported from preliminary clinical trials. Such a promising and original way of curing the disease was developed from positive data obtained in transgenic mice over-expressing the amyloïd protein, although the animal model itself suffers from serious limitations since cognitive performance was probably not significantly altered in such mice;

(ii) The use of " $\beta$-sheet breaker peptides", which could combine with the $\beta \mathrm{A} 4$ peptide to reduce its aggregation because of the role of the $\beta$-sheet structure which contributes to protein insolubility;

(iii) The pharmacological inhibition of $\gamma$-secretase activity;

(iv) The pharmacological modulation of presenilin activity.

Besides vaccination, pharmacological inhibition of the $\gamma$-secretases has now been developed (Roberts 2002). Such an inhibitory procedure could involve some peptides derived from calpaïns, cathepsins or even non peptidic compounds. It is worth noting that a decrease in $\beta \mathrm{A} 4$ biosynthesis could result from the modulation of $\alpha$-secretases contributing to normal processing through pharmacological stimulation of metabotropic glutamate receptors 1 (mGluR1) of the excitatory amino acids (resulting in activation of phospholipase $\mathrm{C}$ signalling pathway) or of the cholinergic muscarinic receptors of the M1 or M3 subtypes.

Regarding the $\beta \mathrm{A} 4$ protein, it has been recently shown in vitro that $\mathrm{Cu}^{2+}$ and $\mathrm{Zn}^{2+}$ ions could promote its aggregation (Bush 2002). Since these two compounds have been shown to accumulate in the brain with age, one could consider that limiting the biodisponibility of the two ions is a possible way of reducing $\beta A 4$ deposits. Such an hypothesis has been investigated in transgenic mice over-expressing APP but the results have not yet been published. Interestingly, however, the controversial antibiotic clioquinol, which has been shown to induce neuromyelopathy in some cases, is known to reduce $\mathrm{Cu}^{2+}$ and $\mathrm{Zn}^{2+}$ brain concentrations.

Experiments still in progress using such compounds have been proposed for Alzheimer's disease but the results again have not yet been published. Other pharmacological compounds, such as desferri oxamine, also known to combine with metals, could have similar properties. So, although no result is presently available, such a strategy should be considered because of the simplicity and possible low cost of apparently interfering with abnormal APP metabolism. Interestingly, in relation to the prion $\operatorname{PrP}$ protein, some very promising results have been obtained in animal models using quinacrine and chlopromazine, which may also act on protein solubility.

Whereas abnormal processing of the tau protein in Alzheimer' disease was more closely correlated to neuronal death there are no significant therapeutic advances in this molecular area. Since tau protein hyperphosphorylation could involve abnormal protein kinase activity (Castro and Martinez 2000), the use of cytochalasins for example, to influence tau protein metabolism through actions at cytosqueleton level could be proposed. In this respect, some protein kinase inhibitors such as staurosporin or compounds acting indirectly on protein phosphorylation such as lithium or benzimidazoles, could exhibit a potential therapeutic effect to be evaluated.

Finally, inflammatory processes, which are a concomitant of brain lesions in Alzheimer's disease, are possibly sensitive to non-steroidian antiinflammatory drugs. Indeed, some authors have concluded that lesion processes in Alzheimer's disease correspond to the chronic inflammatory state in which there are elevated cytokines, specially IL1 and IL6 cytokines. It is presently impossible to evaluate the possible positive influence of such medicine but one could predict that aspirin, indomethacin or ibuprofen could contribute to the reduction of the evolution of the disease. Interestingly, the possible neuroprotective influence of such anti-inflammatory compounds could interfere with cell death processes because of their influence on cyclooxygenase inhibition (Coox-2 inhibitory effects) or on Bax expression, which represent two major steps of the apoptotic processes. 


\section{HOW TO PROMOTE ACTIVE NEUROPROTECTION?}

Neuroprotection is relevant not only for Alzheimer's disease but also for other degenerative diseases resulting either from acute or chronic brain lesions such as in ischemia or various neurodegenerative processes, respectively. Consequently, it is of interest to focus on the cellular and molecular mechanisms of degenerative processes to tentatively limit their effects. Such a research domain is highly investigated but the present knowledge of such processes still remains limited and apoptosis is the most frequent although possibly overestimated hypothesis to explain neuronal death.

\section{Supplementation as an efficient strategy to protect} neurones from degeneration

Even if it might be clearly wrong to say that supplementation is an efficient way to prevent Alzheimer's disease, it is nevertheless possible to propose that some supplementation procedures could reduce the incidence of the disease. Oestrogen therapy (Xu et al. 1998) and more generally the administration of steroid derivatives such as DHEA could represent such a strategy. However, the present results are highly controversial regarding the benefit/risk ratio of these compound administrations. Although still being discussed, data has been produced in support of the claim that the substitutive hormonal treatment for menopause could represent an efficient strategy to delay the evolution of the disease in demented women or even to decrease the age-related risk for a woman to express the disease. One current explanation for possible positive effects on the disease could be related to putative trophic effects of such hormones, as shown during development or in vitro on cellular models. Thus, even when positive, in the context of Alzheimer's disease, the influence of hormonal treatment is certainly non specific.

In the same way, numerous authors have proposed the use of anti-oxidant compounds as neuroprotective cocktails, associating for example, vitamin A (retinol), vitamin $\mathrm{C}$, vitamin $\mathrm{E}$ ( $\alpha$-tocopherol), Q co-enzyme and/or Ginkgo biloba extracts, and also melatonin, super oxide dismutase (SOD)-mimetic agents and other radical oxygen species (ROS) scavengers. These cocktails are aimed at reducing ROS toxicity regarding the possible contribution of oxidative stress to neuronal death (Bains and Shaw 1997). Such an hypothesis is very up to date in the context of an efficient contribution to neuroprotection, more likely in Parkinson's disease. However, because of a possible increase in ROS production in Alzheimer's disease and of the actual non specific stimulatory effects of such compounds, supplementation with anti-oxidant agents could be encouraged since no major side effects have been reported following their administration. But, definitively, anti-oxidants can not be considered as an efficient therapy in Alzheimer's disease. Interestingly, however, selegiline acting as an efficient inhibitor of monoamine oxidase B (IMAO B) was reported to have trophic effects. Ginkgo biloba derivatives could also have a positive influence on patients, possibly through an increase in membranar fluidity and facilitating intercellular communications in the brain. However, it has been shown that the Ginkgo biloba derivative $E g b 761$ exhibits actual free radical scavenging and anti-oxidant properties. Further, such a compound showed neuronal protection, at least in animal models of acute neurodegenerative diseases such as ischemia. Although promising, the results in patients have to be confirmed (DeFeudis 1998). Finally, acting on an elevated level of homocystein through vitamin B (B6, B12) administration could also contribute to decrease the risk factor for the disease, as well as statin administrations for reducing hyper cholesterolemia. But in that case the improvement in the situation could result indirectly from a decrease in heart and vascular diseases, which have been claimed to contribute to an increase in the risk of Alzheimer's disease.

\section{Neuroprotection against "excitotoxicity"}

An excess of excitatory transmission involving excitatory amino acids (EAA) and especially glutamate, which represents one of the major brain neurotransmitters, is thought to be involved in some of the neurodegenerative diseases such as Huntington's chorea, amyotrophic lateral sclerosis (ALS) and even Parkinson's disease. The involvement of EAA is, however, more documented in acute brain lesions resulting from ischemia, anoxia or trauma events. The involvement of such an over-excitatory process is considered either as a causal step of neuronal death or consecutive to the lesion. Indeed, in some cases, an altered glutamatergic transmission could result in an increase in the extracellular glutamate level, reaching toxic concentrations. Alternatively, an excess of the glutamate extracellular level could result from disrupted membranar processes, especially in an anoxic situation.

In Alzheimer's disease, the implication of EAA is far from being proved. However, it is interesting to note in the disease the apparent special susceptibility of the glutamatergic neurones, which represent the major population of hippocampal and 
cortical neurones degenerating along the pathological process. In this respect, a better understanding of the neurodegenerative process of the disease reaching such glutamatergic neurones would give some information about putative strategies to protect the hippocampus and cortical areas from degeneration. We have presently failed to develop efficient neuroprotective strategies to act on excitotoxicity, except with the antiglutamate compound riluzole which has been shown to delay ALS evolution (Doble 1999) or even Parkinson's disease. However, it is to be emphasised that decreasing brain excitability could result from reinforced inhibitory processes, which are known to involve primarily GABAergic neurotransmission. Consequently, direct or indirect GABA agonists could be considered as neuroprotective agents regarding excessive excitatory transmission. In this respect, numerous compounds such as propofol, felbamate, and valproïc acid would be of interest in limiting neurodegenerative processes but primarily in acute diseases.

It is however worth noting that EAA receptor antagonists such as MK801 or ketamine, which block excitatory neurotransmission at the level of NMDA or non-NMDA ionotropic receptors, would theoretically act to reduce excitotoxic processes. Nevertheless, these compounds would also act to limit normal excitatory transmission, which is central in synaptic plasticity, memory and cognitive processes, and further reinforce the behavioural deficits resulting from the disease. In this context, it is interesting to note the recent introduction of memantine, another non competitive NMDA receptor antagonist, which was shown to improve the clinical deficit especially in the most severe forms of Alzheimer's disease.

Recent evidence that interfering with another class of EAA receptors, namely the metabotropic receptor subtypes (mGluR), could also have neuroprotective effects, is very promising and could actually provide new therapeutic strategies in Alzheimer's disease. For example, MPEP, acting as a mGluR5 EAA receptor antagonist, was shown to exhibit neuroprotective actions both in vitro and in vivo (Gasparini et al. 1999). Similarly, mGluR2/3 agonists could exhibit neuroprotective properties. In the latter situation, however, the positive effects could be indirect since the pharmacological stimulation of these receptors has been shown to increase trophic factor production from astrocytes. So, even if we have presently no data in experimental models for Alzheimer's disease, influencing EAA transmission at the mGluR site could represent an unexpected strategy to reduce brain lesion.

Finally, there are two more points to emphasise regarding the putative excitotoxic influence of EAA in Alzheimer's disease. The first point is related to putative EAA-induced apoptosis, which is likely to involve ionised calcium. Many authors have suggested using anti-calcic compounds such as nimodipine to reduce neuronal death. However, little is known about its putative effects in Alzheimer's disease. The use of such compounds is nevertheless worth considering in the light of possible peripheral side effects, especially at heart level. Moreover, limiting calcium intracellular concentrations would theoretically interfere with the role of calcium in physiologic processes such as active secretion of the neurotransmitters involving exocytosis processes. We have, however, to keep in mind that, in fact, only the NMDA receptors are both ligand- and voltage-dependent receptors, the activation of which induces a massive increase in intracellular ionised calcium concentration. Consequently, limiting intracellular calcium concentration will represent an efficient way to reduce NMDA receptor-related neurotransmission, especially when they exhibit hyperactivity. Unfortunately, however, central nicotinic receptors, which are involved in cognitive functions, are also associated with calcium conductance and limiting intracellular calcium concentrations could indirectly affect such cognitive processes.

The second point is related to the role of co-transmitter of EAA played by adenosine, which could act as an endogenous neuroprotector. Indeed, adenosine can represent a co-transmitter released together with EAA from certain nerve terminals. Adenosine was shown to act both at pre-synaptic and post-synaptic levels. At the pre-synaptic level, adenosine can limit the release of EAA by means of pre-synaptic receptors and further decrease their action at the level of the NMDA receptor to increase deleterious calcium influx. The second mechanism involves post-synaptic receptors sensitive to adenosine, so-called purinergic receptors of the A1/A2 subtypes. Consequently, acting to block adenosine inactivation by inhibiting the adenosine uptake process, for example, could contribute to an increase in such an endogenous inhibitory process of excitatory transmission; similarly, activation of the purinergic receptors could have similar neuroprotective effects. In vitro there is some experimental evidence for the efficiency of such a process involving adenosine modulatory action but we are still lacking in vivo evidence for the neuroprotective effect of adenosine.

Regarding excitotoxicity there is consistent evidence for a key contribution in some neurodegenerative diseases for EAA transporters, which are involved in EAA synaptic inactivation,. Rothstein and co-workers (Rothstein et al. 1996) have for a long time suggested the contribution of a deficit in the uptake process of EAA in certain 
forms of ALS following the molecular cloning of the three main EAA transporter subtypes known as EAAT1, EAAT2 and EAAT3 for the 3 main subtypes. Numerous data have more recently confirmed the toxicity of synaptic glutamate, which represents the main EAA neurotransmitter. Consequently, altering glutamate uptake could have major consequences for brain integrity and stimulating the elimination of the synaptic neurotransmitter could actually contribute to neuroprotection. Unfortunately, pharmacological approaches presently available only contribute to reduce EAA uptake. Recent studies, however, suggest that EAA transport is highly sensitive to regulatory processes. Such regulatory processes have been shown to involve both traductional and posttranscriptional mechanisms. In the last case glutamate transport was shown to be regulated through phosphorylation processes primarily involving protein kinase $\mathrm{A}$ and $\mathrm{C}$, depending on the subtype of EAAT considered. It is therefore possible to act on protein kinase activity to increase in some conditions the glutamate uptake and, consequently, its putative neurotoxic effects.

Recent advances in the knowledge of EAA synapse have led to the proposal of another possible mechanism for explaining some forms of neuronal death resulting from the alteration of EAA uptake. Different subtypes of EAAT have been shown to have specific cellular localisation. Moreover, there is also a relative tissue-specificity. For example, EAAT1 (also known as GLAST) is selectively expressed in astrocytes and mainly at the cerebellar level. Similarly, EAAT2, which is called GLT1, is also expressed in astrocytes, and is detected in numerous parts of the forebrain and especially at the cortical and hippocampal levels. Conversely, EAAT3 (EAAC1) is expressed in neurones and not in astrocytes, possibly primarily by post-synaptic targets of EAA nerve terminals and not at presynaptic level.

Data has shown in astrocytes in vitro that the pharmacological inhibition of glutamate uptake resulted in cellular death. In this case, the astrocytes were shown to be affected by a cellular process, which is different from excitotoxicity since pharmacological blockade of EAA receptors did not influence the ability of glutamate inhibition to kill the astrocytes. Further analysis suggests that, in that situation, the degeneration of astrocytes following inhibition of glutamate transport involved oxidative processes related to ROS production. Indeed, glutamate intracellular depletion resulted in astrocytes in a depletion of glutathione, which normally acts in the cell as an anti-oxidant to reduce ROS production. In such a situation the EAATs could no more be considered as central in the neurodegenerative process. More likely the neuronal death could be the consequence of a primary alteration of astrocytes caused by glutamate intracellular depletion (Ré et al. 2004). Consequently, if the astrocytes are no longer able to eliminate the excess of synaptic glutamate, such glutamate could likely contribute to excitotoxicity for altering neuronal survival (Had-Aissouni et al. 2002). If such a mechanism is relevant, neuroprotection has to focus not only on the pharmacological blockade of the NMDA receptors or on resulting calcium influx, but also on favouring the action of glutamate in astrocytes. Such a proposal is rather new and somewhat paradoxical. Interestingly, some specific neuronal populations could also share the properties of astrocytes in being sensitive to glutamate intracellular depletion. This concept is to be further developed.

\section{Neuroprotection and trophic factors}

Trophic factors are presently considered to represent a major hope in limiting neurodegenerative processes (Gao et al. 1997). Recent advances in this field show that such neurotrophic factors could have a certain selectivity in promoting the development and survival of selected brain neuronal populations; for example, brain derived neuronal factor (BDNF) or different neurotrophins have been shown to act primarily on the dopaminergic neurones and the nerve growth factor (NGF) could influence cholinergic neurones rather selectively. However, the situation is probably much more complex and studies are presently aimed at identifying possible new neurotrophic factors as well as receptor subtypes relaying the cellular effects of these factors. Consequently, the proposal to promote neurotrophic protective effects in neurodegenerative diseases would actually represent an efficient means of improving survival or rescuing some neuronal populations involved in such diseases.

However, one of the major problems with this proposal is the consideration of the peptidic nature of the neurotropic factors, which is incompatible with their systemic administration in humans. The challenge is thus to produce non peptidic agonists of these factors, which could enter the brain and represent stable factors regarding the chemical instability of peptides submitted to the influence of peptidases. Another way is to promote the development of chemical vectors, which may contribute to the indirect introduction of peptides in the brain. Such a research field is very active but data is still limited. Interestingly, some tyrosine kinase B receptor (TrKB) agonists or glial derived neuronal factor (GDNF) analogs, such as neurturin, are promising in animal models. Similarly, in vivo 
transplantation of in vitro engineered cells producing trophic factors is still in progress.

An alternative strategy could be to promote the production of trophic factors from cells naturally involved in their biosynthesis, such as astrocytes. Indeed, it was recently suggested that the pharmacological stimulation of the EAA receptors mGluR could actually contribute to improved trophic factor production. Some modulators of EAA receptors such as AMPAkines could have similar effects, as well as certain neuropeptides or analogs. For example, the vasoactive intestinal polypeptide (VIP) was shown to stimulate the production of different neurotrophic factors. Interestingly, lithium itself was shown to exhibit similar properties. Lithium administration was indeed shown in animal models to increase BDNF expression as well as TrkB receptors. Such a strategy to promote neurotrophic factor synthesis is aimed at focussing on glial cells, more precisely on astrocytes, but also on endothelial cells. However, although promising, the data is still very preliminary.

\section{CELLULAR AND GENE THERAPY}

There are presently two current experimental strategies to consider. As proposed above, the first way is to use in vitro engineered cells, eventually immortalised, to produce peptidergic trophic factors or selected deficient proteins to reinforce their endogenous influence. This would be the case for trophic factors but one can consider that such a strategy could also be aimed at producing for example antibodies for reducing deleterious influences. Indeed, it was recently demonstrated that it is possible to block a myelin inhibitory factor biosynthesis, which normally limits axonal regeneration in motor system, using transplantation of cells transfected to produce antibodies against the inhibitory factor. Such a research strategy is probably of more general interest in focussing on molecular factors still to be characterised (Lesh 2002). Because of the development of cell transfection procedures, such a potential therapeutic approach will probably soon be used in experiments in humans but the problem is to develop selected sources of cells to be transplanted and to develop safe transfection methods because of frequent use of viruses for gene transfer.

Another way could be to use stem cells. In this respect, embryonic stem cells could be differentiated in vitro in the ad hoc cells prior to transplantation. In this case, however, the question of the origin of cells and possible immunity limitations is still open. One more ideal possibility would be to use stem cells present in the adult and in the patient himself. The problem is nevertheless to develop a strategy to select proliferation, differentiation and migration of the cells in situ. Regarding Alzheimer's disease it could be proposed, for example, to focus on neurogenesis at the hippocampal level to promote cognitive functions, at least in the earlier stages of the disease.

\section{CONCLUDING REMARKS}

In conclusion, the present proposal to further develop new strategies to improve patients suffering from Alzheimer's disease has no ambition to be exhaustive. Indeed, we have not considered here the possibilities of blocking neuronal death at the level of apoptotic mechanisms using caspases inhibitors (Friedlander 2003) or of stimulating the cAMP signalling pathway, using, for example, phosphodiesterase inhibitors. Moreover, we have not considered the indirect stimulation of cognitive processes using available dopaminergic or serotonergic agonists, which could also contribute to improved behaviour in patients. Our aim was only to emphasise how far it is from the very promising experimental data from experimental neuroscience, to improvement in patients, except in the case of cholinergic transmission stimulation. However, experiments are still in progress and we are actually confident that new data from basic neurobiology will contribute to curing Alzheimer's disease in the near future.

\section{REFERENCES}

Allain H., D. Bentue-Ferrer, O. Trubut, S. Gauthier, B.F. Michel., C. Drieu-La-Rochelle: Alzheimer's disease: the pharmacological pathway. Fund. Clin. Pharmacol. 17: 419-428, 2003.

Bains J.S. and C.A. Shaw: Neurodegenerative disorders in humans: the role of glutathione in oxidative stress-mediated neuronal death. Brain Res. Rev. 25: 335-358, 1997.

Bush A.I.: Metal complexing agents as therapies for Alzheimer's disease. Neurobiol. Aging 23: 1031-1038, 2002.

Castro A. and A. Martinez: Inhibition of tau protein phosphorylation: a new therapeutic strategy for the treatment of Alzheimer's disease and other neurodegenerative disorders. Exp. Opin. Ther. Patents 10: 1519-1527, 2000.

Citron M.: Emerging Alzheimer's disease therapies: inhibition of $\beta$-secretase. Neurobiol. Aging 23: 1017-1022, 2002.

Cutler N.R. and J.J. Sramek: Review of the next generation of Alzheimer's disease therapeutics: 
challenges for drug development. Prog. NeuroPsychopharmacol. Biol. Psychiat. 25: 27-57, 2001.

DeFeudis F.V.: Gingko biloba extract (Egb 761). From chemistry to the clinic. Ullstein Medical, Wiesbaden, pp 401, 1998.

Doble A.: The role of excitotoxicity in neurodegenerative disease: implications for therapy. Pharmacol. Ther. 81: 163-221, 1999

Emilien G., K. Beyreuther, C. Masters, J.M. Maloteaux: Prospects for pharmacological intervention in Alzheimer's disease. Arch Neurol. 57: 454-459, 2000.

Friedlander R.M.: Mechanism of disease: apoptosis and caspases in neurodegenerative diseases. N. Engl. J. Med. 348: 1365-1375, 2003.

Gandy S.: Molecular basis for anti-amyloid therapy in the prevention and treatment of Alzheimer's disease. Neurobiol. Aging 23: 1009-1016, 2002.

Gao W.Q., R.J. Well, M. Dugich-Djordjevic: The therapeutic potentials of neurotrophic factors for diseases of the nervous system. Expert Opin. Ther. Patents 7: 325-338, 1997.

Gasparini F., K. Lingenhohl., N. Stoehr., P.J. Flor, M. Heinrich, L. Vranesic, M. Biollaz, H. Allgeier, R. Heckendorn, S. Urwyler, M.A. Varney, E.C. Johnston, S.D. Hess, S.P. Rao, A.I. Sacaan., E.M. Santori., G. Velicelebi, R. Khun: 2-methyl-6(phenylethynyl)-pyridine (MPEP), a potent, selective and systemically active mGlu5 receptor antagonist. Neuropharmacology 38: 1493-1503, 1999.

Had-Aissouni L., D.B. Ré, A. Nieoullon, L. Kerkerian-LeGoff: Importance of astrocytic inactivation of synaptically released glutamate for cell survival in the central nervous system are astrocytes vulnerable to low intracellular glutamate concentrations? J. Physiol. (Paris) 96: 317-322, 2002.
Hock C., U. Konietzko, J.R. Streffer: Antibodies against beta-amyloid slow cognitive decline in Alzheimer's disease. Neuron 38: 547-554, 2003

Lesh K.P.: Gene transfert to the brain: emerging therapeutic strategy in psychiatry? Biol. Psych. 45: 247-253, 2002.

Nieoullon A.: Bases cellulaires des processus dégénératifs. Thérapie 53: 21-29, 1998.

Ré D.B., J. Boucraut, D. Samuel, S. Birman, L. Kerkerian-LeGoff., L. Had-Aissouni: Glutamate transport alteration triggers differentiation-state selective oxidative death of cultured astrocytes: a mechanism different from excitotoxicity depending on intracellular GSH content. J. Neurochem. 85: 1159-1170, 2003.

Roberts S.B: gamma-secretase inhibitors and Alzheimer's disease. Adv. Drug Delivery Rev. 54: 1579-1588, 2002.

Rothstein J.D., G. Dykes-Hoberg, C.A. Pardo, L.A. Bristol., L. Jin, R.W. Kuncl, Y. Kanai, M.A. Hediger., Y. Wang, J.P. Schielke, F.D. Weltry: Knock out of glutamate transporters reveals a major role for astroglial transport in excitotoxicity and clearance of glutamate. Neuron 16: 675-686, 1996.

Scorer C.A.: Preclinical and clinical challenges in the development of disease-modifying therapies for Alzheimer's disease. Drug Discovery Today 6: 1207-1219, 2001.

Sramek J.J. and N.R. Cutler: Recent developments in the drug treatment of Alzheimer's disease. Drug and Aging 14: 359-373, 1999.

$\mathrm{Xu}$ H., G.K Gouras, J.P. Greenfield: Estrogen reduces neuronal generation of Alzheimer beta-amyloid peptides. Nature Med. 4: 447-451, 1998.

\section{Address:}

André Nieoullon, Cellular Interactions, Neurodegeneration and Neuroplasticity (IC2N) Research Unit UMR6186-CNRS, l’Université de la Méditerranée, 31, Chemin Joseph Aiguier, 13402 Marseilles cedex 20, France; nieoullon@1ncf.cnrs-mrs.fr 